\title{
Interspecific transfer of mammalian artificial chromosomes between farm animals
}

\author{
Filomena Monica Cavaliere • \\ Gian Luca Scoarughi • Carmen Cimmino
}

Received: 10 February 2009 /Revised and Accepted: 29 April 2009/Published online: 23 July 2009

(C) Springer Science + Business Media B.V. 2009

\begin{abstract}
It is often desirable to transfer a mammalian artificial chromosome (MAC) from the cells of one species to those of another. Attempts to carry out such transfer have been successful in some cases and have failed in others. In this study we have tested the hypothesis that centromeric DNA sequence similarity could be a useful criterion for determining MAC host range. Homology studies indicated that the sheep should give positive transfer results. The prediction was tested by introducing into sheep cells a yeast artificial chromosome that contained swine centromeric sequences and that had previously been used to produce a de novo MAC in swine cells. The experiments resulted in the formation of a functional de novo MAC in sheep cells, as attested by FISH analysis. The newly formed MAC remained
\end{abstract}

Both Filomena Monica Cavaliere and Gian Luca Scoarughi contributed equally to this research.

Responsible Editor: Herbert Macgregor.

F. M. Cavaliere $\cdot$ C. Cimmino $(\bowtie)$

Department of Developmental and Cell Biology,

University of Rome 'La Sapienza',

Via dei Sardi, 70,

00185 Rome, Italy

e-mail: carmen.cimmino@uniroma1.it

\section{G. L. Scoarughi}

Department of Public Health Sciences,

University of Rome 'La Sapienza',

P. le Aldo Moro, 5,

00185 Rome, Italy structurally and functionally stable in ovine up to 52 generations. The centromeric sequences present on the newly formed MAC are probably swine sequences, although it cannot be ruled out that some sheep sequences may also have migrated to the MAC. The size of the sheep MAC was determined by atomic force microscopy. Thus, centromeric sequence similarity appears to be a useful criterion for predicting the animal species between which MACs can shuttle.

Keywords sheep $\cdot$ microchromosome . artificial chromosome $\cdot \mathrm{MAC} \cdot \mathrm{SAC}$

$\begin{array}{ll}\text { Abbreviations } \\ \text { AFM } & \text { atomic force microscopy/microscope } \\ \text { CENP-A } & \text { centromere protein A } \\ \text { CHO } & \text { Chinese hamster ovarian cell line } \\ \text { DAPI } & \text { 4',6-diamidino-2-phenylindole } \\ \text { FISH } & \text { fluorescence in-situ hybridization } \\ \text { FITC } & \text { fluorescein isothiocyanate } \\ \text { FLK } & \text { fetal lamb kidney cell line } \\ \text { HAC } & \text { human MAC } \\ \text { MAC } & \text { mammalian artificial chromosome } \\ \text { MIC } & \text { minimal inhibitory concentration } \\ \text { PCR } & \text { polymerase chain reaction } \\ \text { PIEC } & \text { porcine iliac-aortic endothelial cell line } \\ \text { PMBC } & \text { peripheral blood mononuclear cell } \\ \text { SAC } & \text { swine artificial chromosome } \\ \text { WBC } & \text { whole blood cells } \\ \text { YAC } & \text { yeast artificial chromosome }\end{array}$




\section{Introduction}

Much effort has recently been devoted to the use of mammalian artificial chromosome (MAC) vectors as tools in basic and applied fields. The rationale underlying the use of MACs as vector systems is that episomal vectors rely exclusively on the replication and segregation mechanisms of natural chromosomes and may be regarded as relatively safe, since they do not depend on viral expression and do not integrate into the host genome. MACs have a number of additional advantages over other vectors, including capacity for harbouring larger genes, proper control of gene expression, maintenance of gene copy number and absence of side-effects such as immunological responses and cell transformation (Grimes et al. 2001; Conese et al. 2004; Grimes and Monaco 2005; Ehrhardt et al. 2008). MACs can be generated by following two different strategies. One consists in creating greatly shortened derivatives of normal chromosomes, usually through irradiation or telomere sequence-mediated shortening of the large parent chromosome (top-down strategy). Such MACs are linear and contain the sequences necessary for replication and segregation, and must present telomeres at their ends (Lipps et al. 2003; Ren et al. 2006). An alternative bottom-up strategy consists of generating MACs de novo by intracellular assembly of the necessary functional sequences, cloned centromeric DNA and putative replication origins; in this case the presence of telomeric sequences is not required (Harrington et al. 1997; Henning et al. 1999; Ebersole et al. 2000; Kaname et al. 2005; Suzuki et al. 2006). These structures are probably formed by concatemerization of the input DNA; they are therefore considerably larger than the input material and are most likely to be circular. The sequences that are responsible for proper segregation of MACs (and of chromosomes in general) are centromeric sequences and in circular MACs these sequences also furnish replication origins (Ebersole et al. 2000). The centromeric DNA used for construction of circular MACs provides a site for the formation of a centromere following a process that has much in common with the formation of neocentromeres.

Studies with canonical centromeres and neocentromeres have shown that there is no specific sequence requirement for centromere formation, but that satellite DNA is more efficient in centromere assembly than pericentromeric DNA and even more so than dechromatinized euchromatic DNA (Chueh et al. 2005). Extensive work carried out on centromeric protein-centromeric DNA interactions has shed light on many aspects of centromere formation, which involves three-dimensional interactions and epigenetic processes. It has been shown that the centromeres of de novo MACs bind all known centromeric proteins, including CENP-A, CENP-B and CENP-C (Allshire and Karpen 2008; Gieni et al. 2008; Marshall et al. 2008; Okamoto et al. 2007; Okada et al. 2007). The centromere protein A (CENP-A), a variant protein of histone $\mathrm{H} 3$; binds to the centromeric region early in kinetochore formation. While the histone $\mathrm{H} 3$ gene is nearly invariant, the CENP-A gene has evolved rapidly and shows high divergence among mammals, especially in its N-terminal tail ( $\mathrm{Li}$ and Huang 2008). However, little is known about heterologous interspecies satellite-centromeric protein interactions. In these cases the centromere that will form will be a hybrid structure, in the sense that donor satellite will have to interact with the centromeric proteins of the recipient. Thus, it is not unreasonable to hypothesize that the centromeric proteins of a given animal species will interact with different efficiencies with the centromeric DNAs of other species; therefore, the sequence of satellite DNA is very likely to play an important role in the efficient assembly of a hybrid centromere.

Centromeric DNA varies considerably in composition; however, despite the lack of universal sequence motifs, repeat unit length is remarkably similar among mammals. Typically, eukaryotic centromeric regions contain repeat units ranging in length from 150-180 to 300-360 bases, approximately the length required to form a single nucleosome (Henikoff et al. 2001; Hall et al. 2003; Plohl et al. 2008).

Attempts to transfer MACs from one species to another have been successful in some cases and have failed in others: human MACs (HACs) have been transferred into mouse and Chinese hamster (Guiducci et al. 1999; Shinohara et al. 2000). A HAC has been recently transferred from a hamster cell line to a mouse embryonic stem cell line (Paulis et al. 2007). An attempt to transfer a human minichromosome (MC1) into swine cells was unsuccessful (P. Fradiani, personal communication, 2002), as well as the attempt to transfer a swine microchromosome into human cell lines (unpublished data). 
Our interest is to produce transgenic animals that can make important contributions to fields such as the genetic improvement of livestock and the production of pharmaceuticals using animal bioreactors, xenotransplantation, as well as to utilize them as model systems for gene therapy. MAC construction requires much effort and is time consuming. Shuttling of MACs between animal species will greatly enhance the usefulness of artificial chromosome technology: once a MAC is available for use in one animal species, much time and effort would be saved if that MAC could be introduced into other animals.

In this study the homology between pig satellite DNA and the satellite DNA of a number of other mammals was determined in an attempt to shed light on the problem of interspecies MAC transfer. The DNAs examined were from animals in which MAC transfer from pig had failed, and from animals that had not been tested. The homology results were consistent with lack of transfer in previous work and indicated that sheep and to a lesser extent, bovines, should give positive transfer results according to the hypothesis. The prediction was tested by attempting to transfer a de novo MAC (microchromosome) that had been produced in swine cells into sheep cells. A sheep MAC containing swine satellite sequences was in fact obtained and remained structurally and functionally stable in sheep up to 52 generations.

\section{Materials and methods}

Database searching of satellite sequences

and sequence alignment

Satellite and centromeric sequences were obtained from the Entrez nucleotide database by accession number or by keyword search (http://www.ncbi.nlm. nih.gov/entrez/query.fcgi? $\mathrm{db}=$ Nucleotide). The DNA sequences considered in this study were the swine centromeric sequences previously used in SAC construction (accession nos. X62138.1, X62139.1 and X62140.1; Poggiali et al. 2002); a human alphasatellite sequence (accession no. AJ717298); sheep satellite I and satellite II sequences (accession nos. Z17215 e X03117, respectively); a bovine satellite DNA sequence (accession no. M36668); and a mouse consensus DNA sequence derived from a multiple alignment of 19 minor satellite variants (accession nos. from Z22152 to Z22170). For local optimal sequence alignment we used the program LALIGN, an implementation of the SIM algorithm (Huang et al. 1990; Pearson 1997), which assigns a quality score to each possible alignment between two sequences. For multiple sequence alignment the Multalin software was used (Corpet 1988).

The swine artificial chromosome (SAC) precursor

Yeast artificial chromosome (YAC) d1Neo2, enclosing swine centromeric sequences, is contained in the humanized yeast strain $\mathrm{d} 1$ and was derived from the YAC 225D04, which is almost entirely comprised of centromeric sequences (Rogel-Gaillard et al. 1997). YAC d1Neo2 gave rise to the SAC d1Neo2 in PK15 swine cells (Poggiali et al. 2002). The YAC is maintained in $\mathrm{d} 1$ by growth on Ymin medium lacking uracil and lysine.

\section{Cell lines}

The swine cell line PK15 (porcine kidney cell line) was cultured in low-glucose DMEM medium supplemented with $10 \%$ fetal calf serum, $2 \mathrm{mM}$ l-glutamine, $50 \mathrm{U} / \mathrm{ml}$ penicillin and $50 \mathrm{mg} / \mathrm{ml}$ streptomycin (Gibco BRL, Gaithersburg, MD). The sheep cell line FLK (fetal lamb kidney cell line) and the swine cell line PIEC (porcine iliac-aortic endothelial cell line) were cultured in human endothelial medium supplemented with $10 \%$ fetal calf serum, $2 \mathrm{mM}$ l-glutamine, $50 \mathrm{U} /$ $\mathrm{ml}$ penicillin and $50 \mathrm{mg} / \mathrm{ml}$ streptomycin. The Chinese hamster cell line $\mathrm{CHO}$ (Chinese hamster ovarian cell line) was cultured in high-glucose DMEM medium supplemented with $10 \%$ fetal calf serum, $2 \mathrm{mM}$ l-glutamine, $50 \mathrm{U} / \mathrm{ml}$ penicillin and $50 \mathrm{mg} / \mathrm{ml}$ streptomycin (Gibco BRL). All mammalian cell lines were grown at $37^{\circ} \mathrm{C}, 5 \% \mathrm{CO}_{2}, 95 \%$ humidity.

Transfer of YAC into sheep and swine cells

For the transfection of YAC DNA (SAC precursor) into sheep and swine cells, LMP-agarose plugs embedding YAC d1Neo2 yeast cells were fused and $\beta$-agarase digested. The resulting DNA solution was diluted with serum- and antibiotic-free Opti-MEM medium and lipofected into FLK cells and into PK15 cells as control by Lipofectamine 
2000 reagent (Invitrogen Corporation, Carlsbad, CA), following the manufacturer's guidelines. FLK and PK15 cells containing MACs were selected in lowglucose DMEM media containing $500 \mu \mathrm{g} / \mathrm{ml} \mathrm{G} 418$ (Life Technologies, Rockville, MD).

Genomic DNA extraction, PCR primers, sequencing and FISH probes

Genomic DNA extraction was performed both on blood samples and cell culture lines by standard methods (Wizard Genomic DNA purification, Promega, Madison, WI). Additional freeze-thawing lysis steps were added for genomic DNA extraction from cell culture, as suggested by the manufacturer. Mammalian genomic DNAs were isolated from the following sources: PK15 and PIEC cells for pig genomic DNA, FLK cells and lamb blood for sheep genomic DNA, CHO cells for Chinese hamster genomic DNA, PMBC (peripheral blood mononuclear cells) for human genomic DNA, WBC (whole blood cells) for bovine genomic DNA, and a commercial source for mouse genomic DNA (Mouse Genomic DNA, Promega).

PCR amplification of genomic DNAs was performed using pig centromeric primers (SSCSR2 primer pair; SSCSR2A: 5'-agcgcttgcctagttctcacctagc-3'; SSCSR2B: 5'-atcctgagccaagcggcattgg-3'; Miller et al. 1993), sheep satellite I primers (Sat-I L/R primer pair; Sat-I L: 5'ggcaggtgctctcgacttac-3'; Sat-I R: 5'-catgtcagcactgga gagga-3'), and sheep satellite II primers (Sat-II L/R primer pair; Sat-II L: 5'-agaacctgcctcacacacgtagctt-3'; Sat-II R: 5'-actagccetttccttcgctcacacag-3'). SSCSR2 primer pair amplifies a $340 \mathrm{bp}$ pig centromeric fragment with both PK15 and PIEC DNA as template, under the following conditions: $96^{\circ} \mathrm{C}$ for $5 \mathrm{~min} ; 30$ cycles of $94^{\circ} \mathrm{C}$ for $1 \mathrm{~min}, 50^{\circ} \mathrm{C}$ for $1 \mathrm{~min}, 72^{\circ} \mathrm{C}$ for $1 \mathrm{~min} 30 \mathrm{~s} ; 72^{\circ} \mathrm{C}$ for $10 \mathrm{~min}$. The Sat-I primer pair amplifies a $181 \mathrm{bp}$ sheep satellite fragment with FLK DNA as a template, under the following conditions: $96^{\circ} \mathrm{C}$ for $5 \mathrm{~min}$; 30 cycles of $94^{\circ} \mathrm{C}$ for $1 \mathrm{~min}, 57^{\circ} \mathrm{C}$ for $1 \mathrm{~min}, 72^{\circ} \mathrm{C}$ for $1 \min 30 \mathrm{~s}$; $72^{\circ} \mathrm{C}$ for $5 \mathrm{~min}$. The Sat-II primer pair amplifies a 573 bp sheep satellite fragment with FLK DNA as a template, under the following conditions: $96^{\circ} \mathrm{C}$ for $5 \mathrm{~min} ; 30$ cycles of $94^{\circ} \mathrm{C}$ for $1 \mathrm{~min}, 57^{\circ} \mathrm{C}$ for $1 \mathrm{~min}$, $72^{\circ} \mathrm{C}$ for $1 \mathrm{~min} 30 \mathrm{~s} ; 72^{\circ} \mathrm{C}$ for $5 \mathrm{~min}$. Amplicon sequencing was carried out by an automated DNA sequencing service based on fluorescent dye terminator (Bio-Fab Research srl, Pomezia, Italy).
The probes used for FISH analysis were (a) a swine centromeric probe generated by PCR amplification of swine total genomic DNA by the SSCSR2 primer pair; (b) two sheep centromeric probes generated by PCR amplification of sheep total genomic DNA by the Sat-I and Sat-II primer pairs.

\section{Fluorescence in situ hybridization (FISH)}

In situ hybridization was carried out by standard techniques. Cultured cells grown to $60 \%$ confluence in $100 \mathrm{~mm}$ plates were incubated in fresh medium containing $2 \mu \mathrm{g} / \mathrm{ml}$ colcemid (Roche, Mannheim, Germany) for $4 \mathrm{~h}$ and then treated according to standard procedures with $0.075 \mathrm{M} \mathrm{KCl}$ and fixed in methanol-acetic acid (3:1). Artificial chromosomes were detected in the presence of $15 \mu \mathrm{g} / \mu \mathrm{l}$ of salmon sperm DNA with a biotin-labelled pCG probe obtained by nick translation (pCGS990meganeo probe of Poggiali et al. 2002) in both FLK clones and PK15 control clones. This probe is specific for the MAC and corresponds to the left arm of the original YAC $\mathrm{d} 1 \mathrm{Neo} 2$. It was revealed using the sandwich method with avidin-fluorescein isothiocyanate (FITC) and biotinylated anti-avidin (Vector Laboratories, Burlingame, CA). Chromosomes were detected by digoxigenin-labelled centromeric probes using a sheep monoclonal anti-digoxigenin rhodamine conjugate antibody followed by rabbit antisheep IgG antibody Texas red conjugate (Vector) and a final layer of goat anti-rabbit IgG antibody Texas red conjugate (Vector) for increasing sensitivity. Hybridizations were performed under lowstringency conditions $\left(42^{\circ} \mathrm{C}\right)$. DNA was counterstained with DAPI and the slides were mounted using Vectashield mounting medium (Vector). Detection and microscopic analysis of metaphase slides were performed with a Nikon fluorescence microscope.

Atomic force microscopy (AFM)

Metaphase spreads were analysed by using a home-built atomic force microscope described in detail elsewhere (Cricenti and Generosi 1995). The AFM measurements were performed at room temperature, in constant relative humidity $(30 \%)$. The measurements were performed in the weak repulsive regime of constant force with a probe force below 
$1 \mathrm{nN}$ from zero cantilever deflection. The highresolution images were collected at a scanning speed of about 3-4 s/row. The reproducibility of data, including the absence of sample damage or alteration due to the measurement procedure, was carefully tested.

\section{Results}

Mammalian centromeric sequence homology

A search in public databases was made to identify centromeric satellite sequences of the mammalian species considered in this study. The DNA sequences considered were the swine centromeric sequences previously used for the construction of a SAC (Poggiali et al. 2002), a human alpha-satellite sequence, sheep satellite I and II sequences, a bovine satellite DNA sequence, and the mouse minor satellite sequence. These sequences were compared using the local alignment algorithm LALIGN: in the case of repetitive sequences it is appropriate to use a strategy that compares them pairwise and locates local alignment. Such local alignment consists of related sequence regions surrounded by totally unrelated regions (see Materials and Methods). The results of this study are given in Table 1: the score for humanmouse sequence comparison is high, and human MACs have been transferred to mouse, whereas in the case of transfer experiments that in the past have failed, the scores are low. Other species combinations that give high scores are pig-sheep Sat II, pig-bovine, and sheep Sat II-bovine. The same centromeric sequences were also compared globally using the Multalin program (Corpet 1988). The results of this analysis are given in Fig. 1. By this approach it was possible to generate a phenogram depicting sequence relationships among all the satellites considered here. Again, the same two families of close sequence relationship can be defined: in fact the phenogram shows that the pig, bovine and sheep sequences make up a family of sequences that are related more closely to each other than to human and mouse sequences. On the basis of these results and considering our working hypothesis we were encouraged to attempt transfer of a MAC from pig into what appeared to be most likely recipient animal, the sheep.

SAC precursor transfer into sheep cells

On the basis of the centromeric sequence homology results obtained we decided to transfer into fetal lamb kidney cells (FLK) the YAC precursor of the swine MAC d1Neo2 previously assembled by us and used to generate a SAC (Poggiali et al. 2002). In this manner the ability of swine centromeric sequences to drive MAC formation in a non-swine mammalian cell line could be evaluated. Initially the susceptibility of FLK cells to $\mathrm{G} 418(\mathrm{MIC}=750 \mu \mathrm{g} / \mathrm{ml})$ was verified. To transfer the total genomic DNA of the yeast strain AB1380/YAC d1Neo2, agarose plugs embedding AB1380/YAC d1Neo2 spheroplasts were melted, $\beta$-agarase digested and lipofected into FLK cells, as well as PK15 cells as a control.

Various G418-resistant clones were obtained both for sheep FLK cells and control PK15 cells. The positive clones were maintained for over 6 months

Table 1 Sequence comparison analysis: scores were obtained by local optimal sequence alignment carried out by using the program LALIGN. Satellite and centromeric sequences were obtained as described in Materials and Methods

\begin{tabular}{|c|c|c|c|c|c|}
\hline & Pig cen. & Sheep Sat-I & Sheep Sat-II & Bovine sat. & Human $\alpha$-sat. \\
\hline Sheep Sat-I & $56^{\mathrm{a}}$ & & & & \\
\hline Sheep Sat-II & $85^{\mathrm{a}}$ & 77 & & & \\
\hline Bovine sat. & $75^{\mathrm{b}}$ & $67^{\mathrm{b}}$ & $91^{\mathrm{b}}$ & & \\
\hline Human $\alpha$-sat & $48^{\mathrm{c}}$ & $63^{\mathrm{b}}$ & $53^{\mathrm{b}}$ & $57^{\mathrm{a}}$ & \\
\hline Mouse minor sat. & $56^{\mathrm{b}}$ & $49^{\mathrm{b}}$ & $38^{\mathrm{b}}$ & $38^{\mathrm{b}}$ & $74^{\mathrm{a}}$ \\
\hline
\end{tabular}

Pig cen., accession X62139.1; sheep Sat-I, Z17215; sheep Sat-II, X03117; bovine sat., M36668; human $\alpha$-sat., AJ717298; mouse minor sat., consensus sequence derived from 19 variants (Z22151-Z22170).

${ }^{\text {a }}$ Successful MAC transfer.

${ }^{\mathrm{b}} \mathrm{MAC}$ transfer not attempted. ${ }^{\mathrm{c}}$ Failed MAC transfer. 


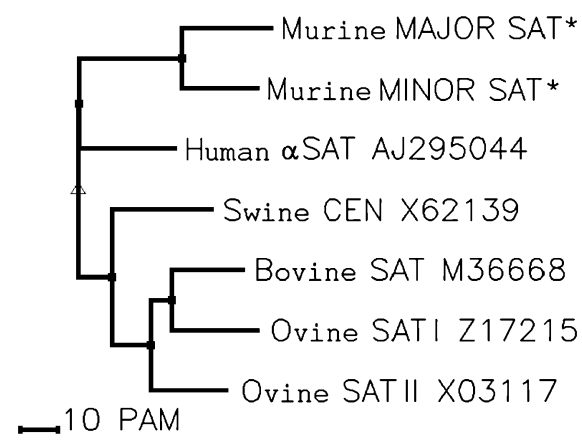

Fig. 1 Phenogram representing a multiple satellite sequence alignment obtained by the Multalin algorithm (Corpet 1988). It depicts sequence relationships among all the satellites considered. PAM, percent accepted mutation. (*) Mouse consensus DNA sequences derived from a multiple alignment of 31 major satellite variants (accession from M32539.1 to M32570.1) and 19 minor satellite variants (accession from Z22152 to Z22170), respectively

under positive selection, after which they were analysed by FISH to determine the presence of a MAC in the lipofected cells.

\section{FISH analysis of G418-resistant FLK clones}

Preliminary experiments were performed to define the primers necessary for generating probes useful for FISH analysis. For the pig centromeric primers we made use of the SSCSR2 primer pair (Miller et al. 1993), which amplifies a 340 bp pig centromeric repeat. For sheep we designed two pairs of primers on sheep satellite I and II DNA (the Sat-I and Sat-II primer pairs). The SSCSR2 primers amplified an amplicon of apparently identical mobility, with pig, sheep and bovine genomic DNA as template (Fig. 2a). Sheep gave a stronger amplification signal than bovine DNA. The amplicons obtained by this PCR assay were sequenced for both strands, and the sequences obtained were compared using the LALIGN algorithm with the global alignment option. The results of pairwise alignments gave 89.8\% identity in 275 nucleotides between swine and sheep (FLK) genomic DNA, and $90.5 \%$ identity in 285 nucleotides between swine and bovine (WBC) genomic DNA. To confirm these surprising data we repeated the SSCSR2 amplification of sheep genomic DNA using a different DNA extracted from domestic sheep WBC. With this DNA the sequencing and comparisons gave a $97.6 \%$ identity in 295 nucleotides between the two sheep DNAs, and a $91.4 \%$ identity in
290 nucleotides between swine and sheep WBC DNA. The sequencing data also provided confirmation of the PCR results with respect to the size of the fragments amplified by the SSCSR2 primers, which are indeed very similar: 293 in swine, 282 in FLK, 289 in domestic sheep, and 307 in bovines. These data demonstrate that the SSCSR2 primers define a stretch of centromeric DNA very similar in size and of very high homology in animals that show high sequence homology in their centromeric DNA, but not in those that do not.

a

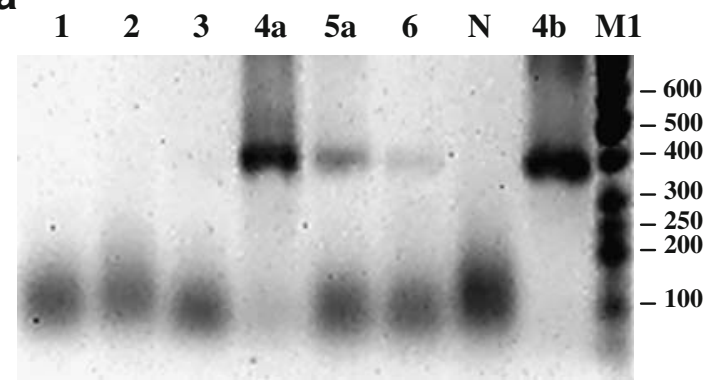

b
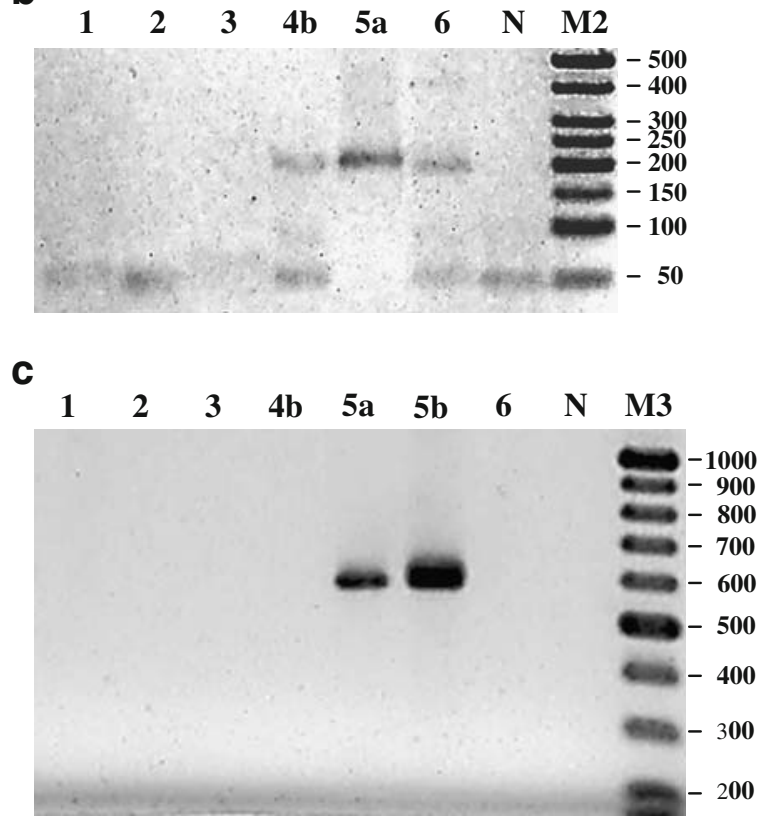

Fig. 2 Gel electrophoresis of PCRs obtained by using SSCSR2 (a), Sat-I (b), and Sat-II (c) oligonucleotides as primers and genomic DNAs obtained from different mammalian cells as template. Lanes: 1, human PMBC; 2, mouse WBC; 3, Chinese hamster $\mathrm{CHO}$; $4 \mathrm{a}$, swine PIEC; 4b, swine PK15;5, ovine FLK; $5 \mathrm{~b}$, lamb WBC; 6 , bovine WBC; N, no template; M1, M2 and M3, markers 
Analogous PCR experiments were performed using Sat-I and Sat-II sheep primer pairs, which amplify sheep centromeric sequences from sheep genomic DNA (D’Aiuto et al. 1997). Sheep Sat-I primers amplified amplicons of similar size, both with pig and bovine genomic DNA (Fig. 2b). Figure 2c shows the PCR results obtained using the Sat-II primer pair: in this case a positive result was obtained only with sheep genomic DNAs. Thus, the PCR results with the Sat-I primers confirmed the sequence similarity between pig centromeric DNA and both sheep and bovine satellite sequences, as shown by
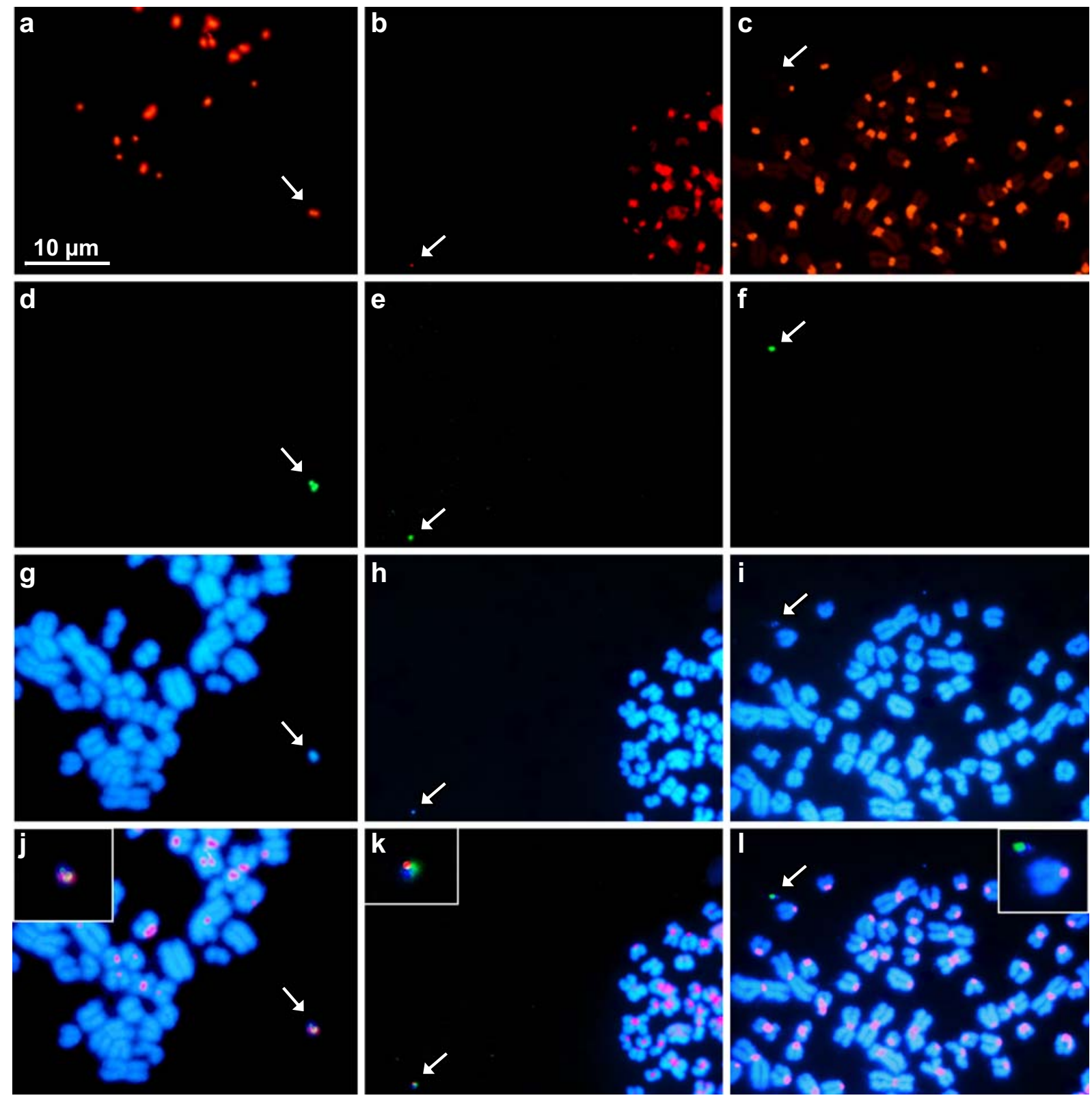

Fig. 3 FISH analysis of the clonal cell line FLKd1Neo2. The white arrows indicate the d1Neo2 MAC. Metaphase chromosomes were hybridized both with centromeric probe $(\mathbf{a}, \mathbf{b}, \mathbf{c})$ and with $\mathrm{pCG}$ probe (d, e, f). Centromeric probes were obtained from PCR amplification of swine total genomic
DNA using SSCR2 primers (a), from PCR amplification of sheep total genomic DNA using Sat-I primers (b) and Sat-II primers (c). Chromosomes were counterstained with DAPI (g, $\mathbf{h}, \mathbf{i})$. Merged images of FISH analysis $(\mathbf{j}, \mathbf{k}, \mathbf{l})$. Inserts show an enlargement of the MAC 
LALIGN and Multalin comparisons, whereas the SatII primers provide a tool for discriminating between sheep satellite and other satellite sequences.

FISH was performed using a pig centromeric probe obtained by PCR amplification of pig total genomic DNA with SSCR2 primers: a strong signal was obtained at the position of the MAC (Fig. 3a), as indicated from the signal obtained with the $\mathrm{pCG}$ probe specific for the MAC and corresponding to the left arm of the original YAC d1Neo2 (Fig. 3d). In principle, this result could be due to the presence of either pig or sheep sequences, or to both. A signal corresponding to the position of the MAC was also obtained using the Sat-I primers that gave crosshybridization under PCR analysis (Fig. 3b). Conversely, no signal was revealed when the Sat-II primers, which are specific for sheep under PCR analysis, were used, strongly indicating that the centromeric sequences present on the MAC are essentially comprised of pig centromeric sequences (Fig. 3c). The above analysis demonstrates the presence of an episomally located MAC in this clone. Only $37 \%$ of the 30 metaphases of the FLK/d1Neo2 clone analysed contained a MAC detectable as overlapping signals with pCG probe and DAPI. However, the MAC is always considerably distant from the grouped chromosomes that make up the spread: like the SAC in PK15/d1Neo2 clones (Poggiali et al. 2002), the MAC showed a propensity to move away from the cluster of chromosomes in metaphase spreads, thus possibly escaping detection. For this reason, the total percentage of revealed MACs is probably an underestimate. MAC copy number per cell was consistently one and no integration events were observed.

Mitotic stability

FLK d1Neo 2 cells were maintained in the presence or absence of G418 and analysed at various times by
FISH to verify MAC extrachromosomal maintenance. FISH analysis was performed after 13 and 52 generations in the absence of selection. The results of these FISH analyses are shown in Table 2 and confirm the functional nature of the MAC centromere. Screening was performed on 30 metaphase spreads for every time point considered and did not show significant differences in the fraction of cells that contained the MAC signal.

Atomic force microscopy analysis of the MAC

In order to determine shape and dimension of the MAC with respect to other sheep natural chromosomes, atomic force microscopy on metaphase spreads was also performed. Three molecules of similar size were found in 10 spreads of FLK d1Neo2 cells, whereas no such molecules were found in FLK control cells. These molecules were thus identified as sheep MACs. In Fig. 4a the white arrow indicates the MAC in proximity of a natural sheep chromosome. The size of this MAC was estimated to be $700 \mathrm{~nm}$ long and $120 \mathrm{~nm}$ high by evaluating the distance between points $A$ and A' (Fig. 4b and d). The MAC was compared with the chromosome identified as autosomal chromosome 2 (Fig. 4c and e), whose length was estimated to be $10500 \mathrm{~nm}$ long and $220 \mathrm{~nm}$ high by evaluating the distance between points B and B'. The heights for the MAC and for chromosome 2 were determined along the lengths of the chromosomes from A to A' (Fig. 4d), and from B to B' (Fig. 4e), respectively. Assuming a value of $250 \mathrm{Mb}$, taken from the literature, for chromosome 2 , and taking into account the ratio of 16 between the lengths of the MAC and the autosome, a very rough estimate of the size of the MAC is about $15 \mathrm{Mb}$. This confirms previous observations showing that MACs formed de novo undergo a great increase in size with respect to the input DNA.

Table 2 Analysis of MAC stability through cell generations

\begin{tabular}{lllcc}
\hline Cell line & Generation w/o selection & No. of metaphases analysed & No. of microchromosomes & \% of cells with signal \\
\hline FLK & 0 & 30 & 0 & 0 \\
FLK d1Neo2 & 0 & 30 & 11 & 36.6 \\
FLK d1Neo2 & 13 & 30 & 11 & 36.6 \\
FLK d1Neo2 & 52 & 30 & 9 & 30.0 \\
\hline
\end{tabular}




\section{a}

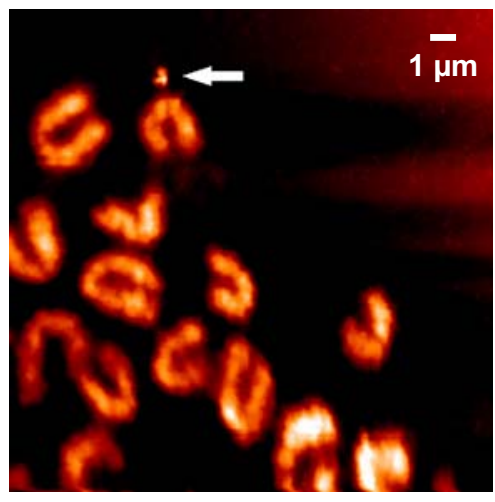

b

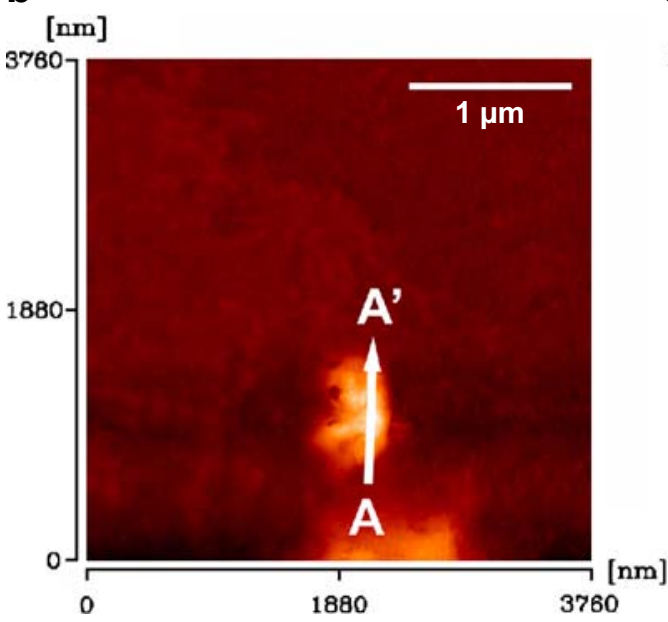

d

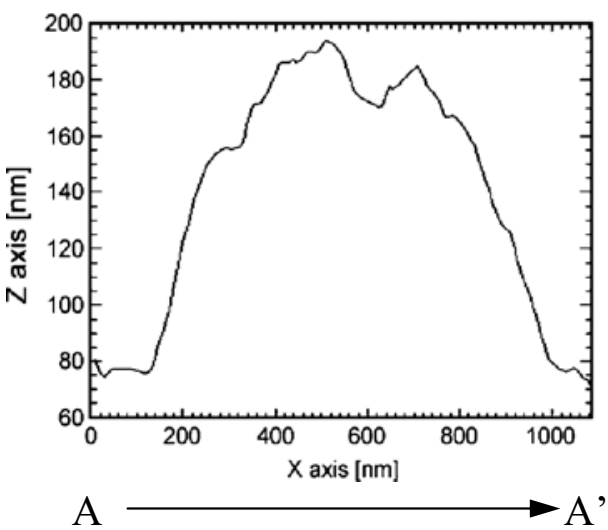

C

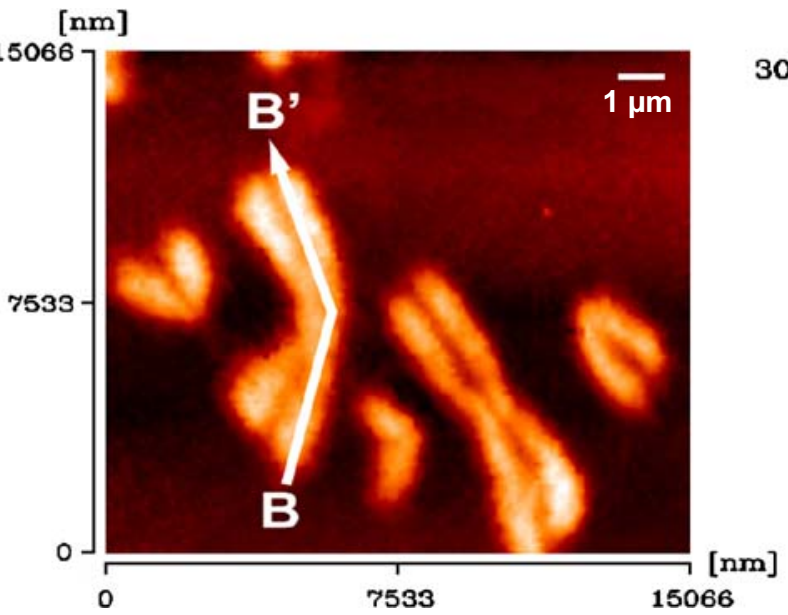

[nm]

06.4

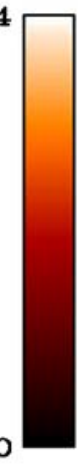

e

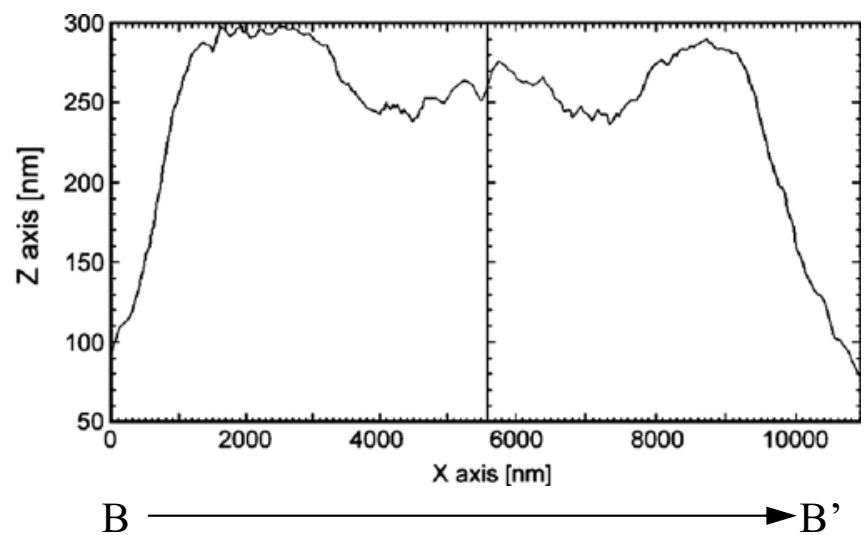

identified as autosomal chromosome 2, whose length was estimated to be $10500 \mathrm{~nm} \pm 200 \mathrm{~nm}$ and height $220 \mathrm{~nm}$, by evaluating the distance between points B and B' (c and e). The heights for the MAC and for chromosome 2 were determined along the lengths of the chromosomes from $\mathrm{A}$ to $A^{\prime}$ (d) and from B to B' (e), respectively

Fig. 4 Atomic force analysis of clonal cell line FLKd1Neo2 metaphase spreads. In (a), the white arrow indicates the MAC in proximity of a natural sheep chromosome. The size of this MAC was estimated to be $700 \mathrm{~nm} \pm 100 \mathrm{~nm}$ long and $120 \mathrm{~nm}$ high by evaluating the distance between points $\mathrm{A}$ and A' (b and d). The MAC was compared to the chromosome 


\section{Discussion}

An aspect of artificial chromosome biology that has not been extensively investigated is that of host range, i.e., the capability of a MAC developed in one animal cell (or animal) to function in the cells of other animals. The present study originated with the construction of a SAC: one of the aims of that study was the hope of using the SAC not only in the pig but also in other animals, including humans. Attempts to transfer the MAC into human, murine and $\mathrm{CHO}$ cells met with failure (unpublished results). This prompted the formulation of the proposition that underlies this study, to wit that, given the presumable central role of centromere sequences in the creation of functional (neo)centromeres, sequence similarity between the DNAs of the donor and recipient centromeres could provide a basis for predicting the likelihood of MAC transfer from one animal species to another. To this end, sequence similarity between pig centromeric DNA and the centromeric DNAs of various mammalian species was searched for in public databases. Two approaches were followed: the local alignment algorithm LALIGN and the Multalin software that produces multiple alignments. The conclusions that could be drawn from both types of analysis were quite similar. The species analysed-pig, sheep, bovine, mouse, and human - could be subdivided into two groups of higher sequence similarity: (a) pig, sheep and bovine; (b) mouse and human. These findings appeared to be in agreement with past experience, since MAC transfer between mouse and human have met with success in the past, whereas attempts to transfer a MAC from pig to mouse or human have failed. The species showing the higher degree of centromeric sequence similarity to the pig was the sheep.

These findings prompted the attempt to transfer into sheep cells the MAC precursor that has been shown to function in swine. The experiments resulted in the formation of functional MACs in sheep cells, as attested by FISH analysis. The sheep MAC was shown to be mitotically stable and its size was determined by atomic force microscopy.

An interesting side-product of the present study is the finding that fragments having a size of about $300 \mathrm{bp}$ and with very high sequence homology one to another are present in the genome of pig, sheep and bovine; these animals comprise one of the two groups of species shown in this study to possess high centromeric sequence similarity.

Thus, centromeric DNA sequence similarity appears to be a useful criterion to determine the possible host range of MACs, making their shuttling between different animals possible. This could be particularly useful in the case of farm animals, since the development of MACs is labour intensive and much time and effort can be saved by developing MACs in one animal species and utilizing them in a number of species that can be identified by the approach outlined in the present study.

Acknowledgements We thank M. Girasole for precious help in carrying out the atomic force microscopy experiments, M. Z. Limongi and S. Di Donna for technical assistance in FISH experiments, and Patrick Chardon for furnishing YAC 225D04B. We are grateful to P. Donini for discussion and comments and for a critical reading of the manuscript. This work was supported by a grant from the MIUR (Ministry of Education, University, and Research, project Modalità alternative di transgenesi animale: ricerche scientifiche sulla costruzione di mini- e microcromosomi artificiali).

\section{References}

Allshire RC, Karpen GH (2008) Epigenetic regulation of centromeric chromatin: old dogs, new tricks? Nat Rev Genet 9:923937

Chueh AC, Wong LH, Wong N, Choo KH (2005) Variable and hierarchical size distribution of L1-retroelement-enriched CENP-A clusters within a functional human neocentromere. Hum Mol Genet 14:85-93

Conese M, Auriche C, Ascenzioni F (2004) Gene therapy progress and prospects: episomally maintained selfreplicating systems. Gene Ther 11:1735-1741

Corpet F (1988) Multiple sequence alignment with hierarchical clustering. Nucleic Acids Res. 16:10881-10890

Cricenti A, Generosi R (1995) Air operating atomic forcescanning tunnelling microscope suitable to study semiconductors, metals and biological samples. Rev Sci Instrum 66:2843

D'Aiuto L, Barsanti P, Mauro S, Cserpan I, Lanave C, Ciccarese S (1997) Physical relationship between satellite I and II DNA in centromeric regions of sheep chromosomes. Chromosome Res 5:375-381

Ebersole TA, Ross A, Clark E et al (2000) Mammalian artificial chromosome formation from circular alphoid input DNA does not require telomere repeats. Hum Mol Genet 9:1623-1631

Ehrhardt A, Haase R, Schepers A, Deutsch MJ, Lipps HJ, Baiker A (2008) Episomal vectors for gene therapy. Curr Gene Ther 8:147-161

Gieni RS, Chan GK, Hendzel MJ (2008) Epigenetics regulate centromere formation and kinetochore function. J Cell Biochem 104:2027-2039 
Grimes BR, Monaco ZL (2005) Artificial and engineered chromosomes: developments and prospects for gene therapy. Chromosoma 114:230-241

Grimes BR, Schindelhauer D, McGill NI, Ross A, Ebersole TA, Cooke HJ (2001) Stable gene expression from a mammalian artificial chromosome. EMBO Rep 2:910-914

Guiducci C, Ascenzioni F, Auriche C, Piccolella E, Guerrini AM, Donini P (1999) Use of a human minichromosome as a cloning and expression vector for mammalian cells. Hum Mol Genet 8:1417-1424

Hall SE, Kettler G, Preuss D (2003) Centromere satellites from Arabidopsis populations: maintenance of conserved and variable domains. Genome Res 13:195-205

Harrington JJ, Van Bokkelen G, Mays RW, Gustashaw K, Willard HF (1997) Formation of de novo centromeres and construction of first-generation human artificial microchromosomes. Nat Genet 15:345-355

Henikoff S, Ahmad K, Malik HS (2001) The centromere paradox: stable inheritance with rapidly evolving DNA. Science 293:1098-1102

Henning KA, Novotny EA, Compton ST, Guan XY, Liu PP, Ashlock MA (1999) Human artificial chromosomes generated by modification of a yeast artificial chromosome containing both human alpha satellite and single-copy DNA sequences. Proc Natl Acad Sci U S A 96:592-597

Huang XQ, Hardison RC, Miller W (1990) A space-efficient algorithm for local similarities. Comput Appl Biosci 6:373-381

Kaname T, McGuigan A, Georghiou A et al (2005) Alphoid DNA from different chromosomes forms de novo minichromosomes with high efficiency. Chromosome Res 13:411-422

Li Y, Huang JF (2008) Identification and molecular evolution of cow CENP-A gene family. Mamm Genome 19:139143

Lipps HJ, Jenke AC, Nehlsen K, Scinteie MF, Stehle IM, Bode J (2003) Chromosome-based vectors for gene therapy. Gene 304:23-33

Marshall OJ, Marshall AT, Choo KH (2008) Three-dimensional localisation of CENP-A suggests a complex higher order structure of centromeric chromatin. J Cell Biol 183:1193-1202
Miller JR, Hindkjaer J, Thomsen PD (1993) A chromosomal basis for the differential organisation of a porcine centromere-specific repeat. Cytogenet Cell Genet 62:3741

Okada T, Ohzeki J, Nakano M et al (2007) CENP-B controls centromere formation depending on the chromatin context. Cell 131:1287-1300

Okamoto Y, Nakano M, Ohzeki J, Larionov V, Masumoto H (2007) A minimal CENP-A core is required for nucleation and maintenance of a functional human centromere. EMBO J 26:1279-1291

Paulis M, Bensi M, Orioli D et al (2007) Transfer of a human chromosomal vector from a hamster cell line to a mouse embryonic stem cell line. Stem Cells 25:2543-2550

Pearson WR (1997) Identifying distantly related protein sequences. Comput Appl Biosci 13:325-332

Plohl M, Luchetti A, Meštrović N, Mantovani B (2008) Satellite DNAs between selfishness and functionality: structure, genomics and evolution of tandem repeats in centromeric (hetero)chromatin. Gene 409:72-82

Poggiali P, Scoarughi GL, Lavitrano M, Donini P, Cimmino C (2002) Construction of a swine artificial chromosome: a novel vector for transgenesis in the pig. Biochimie $84: 1143-1150$

Ren X, Tahimic CG, Katoh M, Kurimasa A, Inoue T, Oshimura M (2006) Human artificial chromosome vectors meet stem cells: new prospects for gene delivery. Stem Cell Rev 2:43-50

Rogel-Gaillard C, Bourgeaux N, Save JC et al (1997) Construction of a swine YAC library allowing an efficient recovery of unique and centromeric repeated sequences. Mamm Genome 8:186-192

Shinohara T, Tomizuka K, Takehara S et al (2000) Stability of transferred human chromosome fragments in cultured cells and in mice. Chromosome Res 8:713-725

Suzuki N, Nishii K, Okazaki T, Ikeno M (2006) Human artificial chromosomes constructed using the bottom-up strategy are stably maintained in mitosis and efficiently transmissible to progeny mice. J Biol Chem 281:2661526623 